Pacific Journal of Mathematics

BRANCHED IMMERSIONS BETWEEN 2-MANIFOLDS O 


\title{
BRANCHED IMMERSIONS BETWEEN 2-MANIFOLDS OF HIGHER TOPOLOGICAL TYPE
}

\author{
John D. Elwin and Donald R. Short
}

In this paper, branched immersions between compact orientable 2-manifolds are considered. Branched immersions are smooth maps whose only singularities are branch points, i.e., points of the domain where the map is locally topologically equivalent to $z \rightarrow z^{r}(r=2,3, \cdots)$. Originally these maps were studied in connection with Douglas' solution to Plateau's problem.

The maps considered here are required to satisfy natural boundary hypothesis which have been motivated by minimal surface studies. The main result completely decides the existence question for a branched immersion between compact orientable 2-manifolds with or without boundary.

I. It has been shown [2] that for a suitable collection of boundary curves, the minimal surface solutions include surfaces of positive genus. It is of interest then to study the existence question for branched immersions between surfaces of higher topological type. Throughout this paper all manifolds considered will be compact orientable 2-manifolds with or without boundary.

The organization of the paper is as follows: §II contains basic definitions and previous results obtained by the authors; \$III is devoted to constructions utilized in the proof of the main theorem which appears in $\S I V$. Let us now state this theorem under the boundary hypothesis restriction of $\S I I$.

Suppose $M$ and $N$ are manifolds of genus $g_{1}$ and $g_{2}$ with $m_{1}$ and $m_{2}$ discs removed respectively. Then

THEOREM 3. There exists a branched immersion $M \rightarrow N$ satisfying boundary conditions (1) if and only if

Case I. $\quad\left(m_{1}=0\right): g_{1}=g_{2}$ or $g_{1} \geqq 2 g_{2}-1$

Case IIa. $\left(m_{1} \neq 0, g_{1} \leqq g_{2}\right): m_{1} \geqq 2\left(g_{2}-g_{1}\right)+m_{2}$

Case IIb. $\left(m_{1} \neq 0, g_{1}>g_{2}\right): m_{1}>m_{2}$.

This result extends those which appear in [4].

II. Basic definitions and previous results necessary for the main theorem are presented in this section. Suppose $f: M \rightarrow N$ is a smooth map between compact orientable 2-manifolds with or without boundaries $\partial M$ and $\partial N$. $p \in M$ is called a branch point 
of order $r-1$ if $f$ is locally topologically equivalent to $z \rightarrow z^{r}$ at $p$. In this work we will assume the following additional boundary conditions:

(i) $\quad \partial N \subset f(\partial M)$

(ii) $f_{\mid \partial M}$ is a homeomorphism.

Since $M$ is a compact 2-manifold, $\partial M$ is a disjoint union of 1 -spheres and by the above assumptions $f(\partial M)$ is a disjoint union of the same number of 1-spheres. Let $B$ denote the set of branch point images of $f$ and let $\gamma(q)$ represent the cardinality of the fiber over $q \in N$. From the local product structure of these maps $\gamma$ is constant on the components $\left\{A_{i}\right\}$ of $N-f(\partial M) \cup B$ and changes by exactly one across any boundary image. The value of $\gamma$ on a boundary image point $q$ is the largest value $\gamma$ attains on the adjacent components $\left\{A_{i}\right\}$ which meet every neighborhood of $q$.

If we denote each boundary image 1-sphere with all of its branch point images removed by $C_{j}$, then $\gamma\left(A_{i}\right)$ and $\gamma\left(C_{j}\right)$ are defined as the constant value $\gamma$ assumes at every point of $A_{i}$ or $C_{j}$. Using this notation we may now state the basic Euler characteristic formula whose proof may be found in [4].

THEOREM 1. Suppose $f: M \rightarrow N$ is a branched immersion between compact orientable 2-manifolds. Assume that $f_{\mid \partial M}$ is a homeomorphism and in addition that $\partial N \subset f(\partial M)$. Then

$$
\chi(M)=\sum_{i} \gamma\left(A_{i}\right) \chi\left(A_{i}\right)-\sum_{j} \gamma\left(C_{j}\right) \chi\left(C_{j}\right)+\sum_{b \in B} \gamma(b)
$$

The most useful form of the above formula may be stated as a corollary which eliminates the need to distinguish between interior and boundary branch point images. An equivalent version of this formula appears in Ahlfors [1]. However, to state this corollary another multiplicity function which incorporates the branching order must be defined. If $q \in N$, define $o(q)$ to be the sum of the orders of the branch points in $f^{-1}(q)$ or zero if $f^{-1}(q)$ contains only regular points. Define $\mu$ at each point $q \in N$ by $\mu(q)=\gamma(q)+o(q)$. From its definition it is immediate that $\mu$ is constant on the topological components $\left\{N_{i}\right\}$ of $N-f(\partial M)$ and on each boundary image 1-sphere.

COROLlaRY 1. Under the hypothesis of Theorem 1

$$
\chi(M)=\sum_{i} \mu\left(N_{i}\right) \chi\left(N_{i}\right)-V
$$

where $V$ denotes the total branching order $\Sigma_{b \in B} o(b)$. 
Proof. Let $B_{i}=N_{i} \cap B$ and let $\left|B_{i}\right|$ denote its cardinality. From these definitions we have $N_{i}=A_{i} \cup B_{i}$ and

$$
\chi\left(N_{i}\right)=\chi\left(A_{i}\right)+\left|B_{i}\right|
$$

Since $A_{i}$ contains only images of regular points

$$
\mu\left(N_{i}\right)=\mu\left(A_{i}\right)=\gamma\left(A_{i}\right) .
$$

Hence

$$
\begin{aligned}
\mu\left(N_{i}\right) \chi\left(N_{i}\right) & =\gamma\left(A_{i}\right) \chi\left(A_{i}\right)+\sum_{b \in B_{i}} \mu(b) \\
& =\gamma\left(A_{i}\right) \chi\left(A_{i}\right)+\sum_{b \in B_{i}} \gamma(b)+\sum_{b \in B_{i}} o(b) .
\end{aligned}
$$

Similarly denoting the $j$ th image 1 -sphere by $S_{j}^{1}$ and $B_{j}=S_{j}^{1} \cap B$ we have $S_{j}^{1}=C_{j} \cup B_{j}$. Since $B_{j}$ is finite

$$
\begin{aligned}
0 & =\chi\left(S_{j}^{1}\right)=\chi\left(C_{j}\right)-\left|B_{j}\right| \\
\mu\left(S_{j}^{1}\right) & =\mu\left(C_{j}\right)=\gamma\left(C_{j}\right)
\end{aligned}
$$

and thus

$$
\begin{aligned}
& 0=\gamma\left(C_{j}\right) \chi\left(C_{j}\right)-\sum_{b \in B_{j}} \mu(b) \\
& 0=\gamma\left(C_{j}\right) \chi\left(C_{j}\right)-\sum_{b \in B_{j}} \gamma(b)-\sum_{b \in B_{j}} o(b) .
\end{aligned}
$$

Substitution of equations (3) and (4) into Theorem 1 completes the proof.

If $\partial M=\partial N=\phi$ we may express the formula in Theorem 1 in terms of the genera of $M$ and $N$. The resulting equation is a generalization of the classical Riemann-Hurwitz formula [5].

Corollary 2. Suppose $f: M \rightarrow N$ is a $n$-sheeted branched covering between compact orientable 2-manifolds without boundary of genus $g_{1}$ and $g_{2}$ respectively. Then

$$
2\left[n\left(1-g_{2}\right)+g_{1}-1\right]=V .
$$


In [4], Theorem 1 was applied to the question of existence or nonexistence of a branched immersion from the 2-sphere with $m$ discs removed onto a compact orientable 2-manifold $N$ of genus $g$. Letting $S_{m}^{2}$ represent the above sphere the main result of the paper is the following:

THEOREM 2. There exists a surjective branched immersion $f: S_{m}^{2} \rightarrow N$ satisfying boundary conditions (1) if and only if $m \geqq 2 g$.

The goal of the present paper is to completely decide the existence question for branched immersions between compact orientable 2manifolds each having any number of discs removed subject to the boundary restrictions (1).

III. This section contains three constructions which will be utilized in the proof of the existence theorem in $\S I V$. Construction 1 is equivalent to the classical Riemann surface construction of cutting $p$ slits in two branches and identifying opposite sides.

Construction 1. Suppose $N$ is a compact 2-manifold with or without boundary. Let $X$ be a submanifold formed by removing from $N$ the interiors of $p$ disjoint homeomorphic images of the disc.

$$
\left.X=N-\vee_{i=1}^{p} \text { (int } D\right) \text {. }
$$

Now form the disjoint union of $p$ copies of the 2-sphere with each parameterized by geodetic co-ordinates. From this disjoint union construct the manifold $Y$ by removing the interior of $n$ identical disjoint discs centered at $(2 \pi j / n, 0)(j=1, \cdots, n)$ from each sphere.

$$
\left.Y=\vee_{i=1}^{p}\left(S^{2}-\bigvee_{j=1}^{n} \text { (int } D\right)\right)
$$

Attach $X$ to $Y$ via a homeomorphism of the boundaries of the removed discs of $N$ with the removed discs centered at $(0,0)$ from each sphere in $Y$. Adjust this homeomorphism by adding $2 \pi / n$ to the longitude. Then using this new map attach another copy of $X$ to $Y$. Proceed in this fashion until $n$ copies of $X$ are attached. Denote the resulting manifold by $M$.

EXAmple 1. Let $N$ be the torus and set $p=3$ and $n=2 . \quad M$ becomes the compact orientable 2-manifold of genus 4 . 


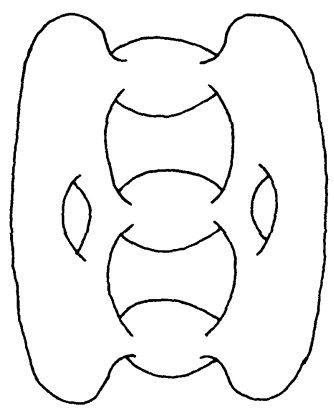

FIGURE 1

The Euler characteristic of $M$ is related to that of $N$ by the formula

$$
\chi(M)=n \chi(N)-2 p(n-1)
$$

LEMMA 1. There exists a surjective branched covering $f: M \rightarrow N$ having $2 p$ branch points each of order $n-1$ and satisfying $\mu(f)=$ $n$. The total branching order is $V=2 p(n-1)$.

Proof. Define $f$ by the formula

$$
f(x)= \begin{cases}\operatorname{incl}(x) & x \in X \\ h_{i}\left(n \theta_{x}, \phi_{x}\right) & x \in Y \text { with geodetic co-ordinates } \\ & \left(\theta_{x}, \phi_{x}\right) \text { in the } i \text { th sphere }\end{cases}
$$

where $h_{i}$ is a homeomorphism of the 2-sphere with the disc at $(0,0)$ removed onto the $i$ th disc removed from $N$.

Construction 2. Suppose $\bar{M}$ is a compact 2-manifold with or without boundary. Let $X$ be a submanifold formed by removing from $\bar{M}$ the interior of a homeomorphic image of a disc

$$
X=\bar{M}-\text { int } D \text {. }
$$

Now suppose $T$ is a torus paramaterized by toriodal co-ordinates. Let $\left\{D_{i}\right\}$ be identical disjoint homeomorphic images of the unit disc centered at $(2 \pi j / n, 0)(j=1, \cdots, n)$. Define $N$ to be the manifold formed by attaching $T$ - int $D_{n}$ to $X$ via a homeomorphism of the boundaries of the removed discs. Now remove the interiors of the remaining discs $\left\{D_{j}\right\}(j=1, \cdots, n-1)$ from $N$. Attach $X$ to $N-\vee_{j=1}^{n-1}$ int $D_{j}$ via the homeomophism used to form $N$ adjusted by $2 \pi / n$ in the longitudinal co-ordinate. Proceed as in Construction $\mathrm{I}$, attaching copies of $X$ to the 
remaining boundary images of the removed discs. Denote this manifold by $M$.

EXAMPLE 2. Let $\bar{M}$ be the double torus and set $n=3 . \quad M$ is then the 2-manifold of genus 7 , and $N$ the manifold of genus 3 .

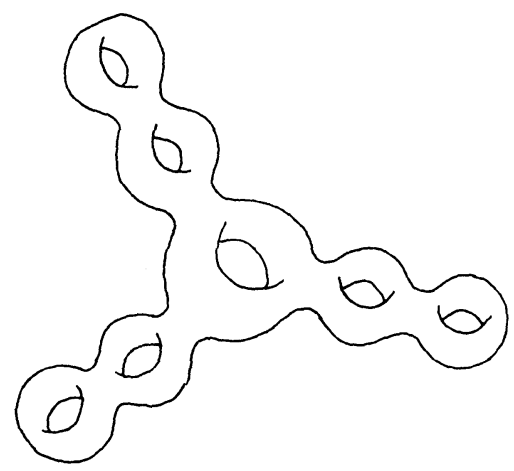

FIGURE 2

The Euler characteristic of $M$ is given by the formula

$$
\chi(M)=n(\chi(\bar{M})-2)=n \chi(N) .
$$

LEMMA 2. There exists a covering projection $f: M \rightarrow N$ having multiplicity $n$.

Proof. Define $f$ by the formula

$$
f(x)= \begin{cases}\operatorname{incl}(x) & x \in X \\ \left(n \theta_{x}, \phi_{x}\right) & \left.x \in T-\vee_{j=1}^{n} \text { (int } D_{j}\right) \text { with } \\ & x=\left(\theta_{x}, \phi_{x}\right) \text { in toroidal } \\ & \text { co-ordinates. }\end{cases}
$$

Construction 3. This construction is a combination of Constructions 1 and 2. Again, let $\bar{M}$ be a compact 2-manifold with or without boundary. Suppose $\left\{D_{i}\right\}(i=0,1, \cdots, p)$ is a set of disjoint homeomorphic images of the disc in $\bar{M}$. As in Construction 2 form $N$ by attaching a handle to the boundary of $D_{0}$. Then proceeding as in 2, attach $n-1$ copies of $M$ - int $D_{0}$ around the handle on $N$. Denote the resulting manifold by $\hat{M}$. Now form the disjoint union of $p$ copies of the 2-sphere each having 2 discs removed. As in construction 1 attach 
$\hat{M}-\vee_{i=1}^{p}\left(\right.$ int $\left.D_{i}\right)$ to one side of these spheres and $N-\vee_{i=1}^{p}\left(\right.$ int $\left.D_{i}\right)$ to the other. Denote this manifold by $M$.

Example 3. Suppose $\bar{M}$ is the torus and set $p=3$ and $n=3$. $\quad M$ is then the 2-manifold of genus 8 , and $N$ the manifold of genus 2 .

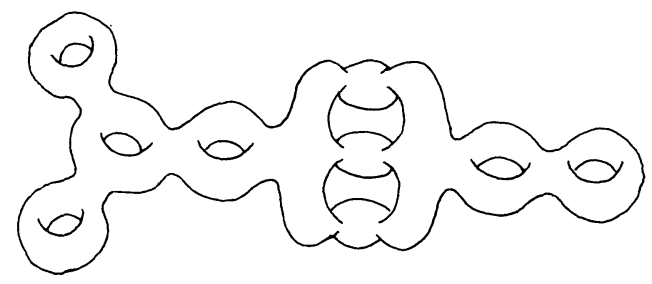

FIGURE 3

The Euler characteristic of $M$ is given by

$$
\chi(M)=n \chi(N)-2 p+\chi(N) \epsilon
$$

where

$$
\epsilon=\left\{\begin{array}{lll}
0 & \text { if } & p=0 \\
1 & \text { if } & p>0
\end{array}\right.
$$

Lemma 3. There exists a surjective branched covering $f: M \rightarrow N$ such that $\mu(f)=n+\epsilon$. f has $2 p$ branch points each of order 1 giving a total branching order $V=2 p$.

This lemma follows by combining the proofs of Lemmas 1 and 2 .

A slight modification of Construction 1 gives the following technical lemma:

Lemma 4. If $D$ is the homeomorphic image of a disc in a 2manifold $M^{\prime}$ then there is a branched covering $f: M^{\prime}-$ int $D \rightarrow M^{\prime}$

Proof. Construction 1 with $p=1, n=2$ and $N=M^{\prime}$ gives a branched covering $f: M \rightarrow M^{\prime}$. Now let $W$ denote the submanifold of $M$ obtained by attaching just one copy of $M^{\prime}-$ int $D$ to $S^{2}-\vee_{j=1}^{2}$ int $D_{j}$ instead of two. Thus we may define $g: W \rightarrow M^{\prime}$ by $f=f \circ$ incl. From the definition of $f$ we know that $g$ is a surjective branched covering. However, $W$ is just $M^{\prime}-$ int $D$ with an attached collar and hence $W$ is homeomorphic to $M^{\prime}$ - int $D$. Thus the composite of $g$ with this homeomorphism is the desired map. 
IV. Let $M$ and $N$ denote compact orientable 2-manifolds of genus $g_{1}$ and $g_{2}$ with $m_{1}$ and $m_{2}$ discs removed respectively. In addition we will require any branched immersion $M \rightarrow N$ to satisfy boundary restrictions (1). Consider the following cases: $I\left(m_{1}=0\right), I I a\left(m_{1} \neq 0\right.$, $\left.g_{1} \leqq g_{2}\right)$, and $I I b\left(m_{1} \neq 0, g_{1}>g_{2}\right)$.

THEOREM 3. There exists a branched immersion $M \rightarrow N$ satisfying boundary conditions (1) if and only if

(Case I). $\quad g_{1}=g_{2}$ or $g_{1} \geqq 2 g_{2}-1$,

(Case IIa $) . \quad m_{1} \geqq 2\left(g_{2}-g_{1}\right)+m_{2}$,

(Case IIb). $m_{1}>m_{2}$.

Proof. Case I $\left(m_{1}=0\right)$. Note that boundary conditions (1) imply $m_{2}=0$. Hence if a branched immersion $M \rightarrow N$ exists it must satisfy Corollary 2 of Theorem 1 which states

$$
V=2\left(n\left(1-g_{2}\right)+g_{1}-1\right) \text {. }
$$

If $n=1$ the total branching order must be zero and hence $g_{1}=$ $g_{2}$. If $n \geqq 2$ we have

$$
n\left(1-g_{2}\right)+g_{1}-1 \geqq 0
$$

or

$$
g_{1} \geqq 1-n\left(1-g_{2}\right) \geqq 2 g_{2}-1 \text {. }
$$

Thus the only possibilities are $g_{1}=g_{2}$ and $g_{1} \geqq 2 g_{2}-1 . \quad g_{1}=g_{2}$ is immediate and thus we need only show a branched immersion exists whenever $g_{1} \geqq 2 g_{2}-1$.

Assume $g_{1}>2 g_{2}-1$. Choose $N$ of Construction 1 to be the compact orientable surface of genus $g_{2}$ and let $n=2$. Under the above assumptions, $p=g_{1}-2 g_{2}+1$, and upon rotation the desired map is obtained. If $g_{1}=2 g_{2}-1\left(g_{2}=0\right.$ is impossible) we may use Construction 2 with $\bar{M}$ the compact orientable surface of genus $g_{2}-1$. In this case $V=0$.

Case IIa. $\quad\left(m_{1} \geqq 2\left(g_{2}-g_{1}\right)+m_{2}\right)$. If $g_{1}<g_{2}$ we can map $M$ onto surfaces of genera $g_{1}+1, \cdots, g_{2}$ by successively stretching and identifying with overlap, pairs of boundaries from $M$. We then have sufficient boundaries remaining to map onto those of $N$. Additional holes in the domain may be mapped into the boundary overlap region. If $g_{1}=g_{2}$ consider the identity map id: $N \rightarrow N$. Let $N_{k}$ denote the manifold 
obtained by removing $k$ discs from $N$. Note that $M=N_{m_{1}-m_{2}}$ and that $N_{0}=N$. Lemma 4 of $\S$ II provides a branched immersion $f_{k}: N_{k} \rightarrow N_{k-1}$. By successively composing the maps $\left\{f_{k}\right\}$ with id, we obtain a branched immersion $M \rightarrow N$.

Assume $m_{1}<2\left(g_{2}-g_{1}\right)+m_{2}$. The above proceedure for $g_{1}<g_{2}$ describes a map $M_{k} \rightarrow M$ where $M_{k}$ has genus $g_{1}-k$ and has $m_{1}+2 k$ discs removed. Note that $M_{g_{1}}$ is the 2-sphere $S^{2}$ with $m_{1}+2 g_{1}$ discs removed. If there exists a map from $M \rightarrow N$ then by composition there must also exist a map from $M_{g_{1}} \rightarrow N$. Theorem 2 states that there exists a branched immersion from the 2-sphere with $m$ discs removed onto a compact orientable 2-manifold without boundary of genus $g$ if and only if $m \geqq 2 g$. We may attach $m_{2}$ discs to the boundary of $N$ and $m_{2}$ discs to the corresponding portion of the boundary in $M_{g_{1}}$ to obtain a map from the 2-sphere with $m_{1}-m_{2}+2 g_{1}$ discs removed onto a 2manifold without boundary. Thus from Theorem 2

$$
m_{1}-m_{2}+2 g_{1} \geqq 2 g_{2}
$$

or

$$
m_{1} \geqq 2 g_{2}-2 g_{1}+m_{2} \text {. }
$$

By assumption the reverse inequality is true and hence no branched immersion $M \rightarrow N$ exists.

Case IIb $\left(m_{1} \neq 0, g_{1}>g_{2}\right)$. For existence, boundary conditions (1) require $m_{1} \geqq m_{2}$. If $m_{1}=m_{2} \neq 0$ we may attach $m_{1}$ discs to both $M$ and $N$ to obtain a covering projection with covering multiplicity one. This implies that $V=0$ and hence $g_{1}=g_{2}$. Therefore, if a branched immersion exists when $g_{1}>g_{2}$ it follows that $m_{1}>m_{2}$.

Initially let us assume $m_{1}=1$ and $m_{2}=0$. As in the proof of Lemma 4 we will use a slight modification of Construction 1 . In the range $g_{1} \geqq 2 g_{2}$ choose $n=2, p=g_{1}+1-2 g_{2}$ and let one copy of $N$ be the target manifold of this theorem and the other copy the target manifold with a disc removed. Note that the image of the above map is a manifold of genus $g_{2}$ covered twice by the map with the exception of the interior of a contractible disc which is covered once. Additional holes may be added simultaneously to the domain and the target by using the portion covered once. Lemma 4 of \$II allows additional holes in the domain and hence for $g_{1} \geqq 2 g_{2}$ the result is proved.

For $g_{1}<2 g_{2}$ we will again use a modification of Construction 1 and initially assume $m_{1}=1$ and $m_{2}=0$. Choose $n=2, p=1$ and let one copy of $N$ in Construction 1 have genus $g_{2}$. Let the other copy have genus $g_{1}-g_{2}$ and have a single disc removed. Upon rotation the 
surface of genus $g_{1}-g_{2}$ will form a "collar" on the surface of genus $g_{2}$. Additional holes in the target or domain may be added in the same fashion as in the case $g_{1} \geqq 2 g_{2}$. An example will clearly illustrate the process.

EXAmple 4. Assume $M$ has genus 5 with 3 discs removed and $N$ has genus 3 with 1 disc removed.

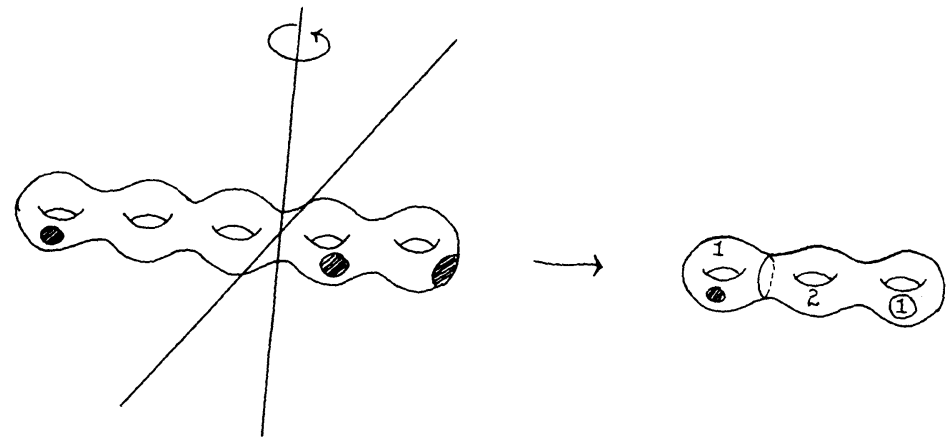

FigURE 4

The following corollary may be obtained as a consequence of the constructions. This corollary states that in the case $g_{2}>0$ and $m_{1}=0$ the formula $V=2\left(\mu(f)\left(1-g_{2}\right)+g_{1}-1\right)$ gives the best possible information on the existence and the nature of branched immersions satisfying our hypotheses.

COROLlaRY. For $g_{2}>0$ and $m_{1}=0$, each choice of total branching order $V$ and covering multiplicitiy $\mu(f)$ which satisfies the formula $V=2\left(\mu(f)\left(1-g_{2}\right)+g_{1}-1\right)$ can be realized by a branched immersion $M \rightarrow N$.

Proof. We have two cases which both use Construction 3. For $V=0$, choose $n=\mu(f), p=0, \epsilon=0$ and let the genus of $\bar{M}$ be $g_{2}-1$. For $V \neq 0$, choose $n=\mu(f)-1, p=V / 2, \epsilon=1$ and again let the genus of $\bar{M}$ be $g_{2}-1$. Construction 3 then gives the desired mapping.

\section{REFERENCES}

1. L. Ahlfors, Zur theorie der Überlagerungflächen, Acta Math. (Uppsala), 65 (1935).

2. R. Courant, Dirichlet's Principle, Interscience Publishers, Inc., London (1950).

3. R. Gulliver, R. Osserman, and H. Royden, A theory of branched immersions of surfaces, Amer.

J. Math., 45 (1973).

4. J. Elwin and D. Short, Branched immersions onto compact orientable surfaces, Pacific J. Math., 49 (1974), (to appear).

5. - A generalized Riemann-Hurwitz formula (to appear).

Received June 12, 1974 and in revised form August 27, 1974. 


\section{PACIFIC JOURNAL OF MATHEMATICS}

\section{EDITORS}

RICHARD ARENS (Managing Editor)

University of California

Los Angeles, California 90024

\section{J. DugundJI}

Department of Mathematics University of Southern California Los Angeles, California 90007

D. Gilbarg and J. Milgram

Stanford University

Stanford, California 94305

\section{ASSOCIATE EDITORS}
E. F. BECKENBACH
B. H. NeumanN
F. WoLF
K. YoshiDA

\section{SUPPORTING INSTITUTIONS}

\author{
UNIVERSITY OF BRITISH COLUMBIA \\ CALIFORNIA INSTITUTE OF TECHNOLOGY \\ UNIVERSITY OF CALIFORNIA \\ MONTANA STATE UNIVERSITY \\ UNIVERSITY OF NEVADA \\ NEW MEXICO STATE UNIVERSITY \\ OREGON STATE UNIVERSITY \\ UNIVERSITY OF OREGON \\ OSAKA UNIVERSITY
}

\author{
UNIVERSITY OF SOUTHERN CALIFORNIA \\ STANFORD UNIVERSITY \\ UNIVERSITY OF TOKYO \\ UNIVERSITY OF UTAH \\ WASHINGTON STATE UNIVERSITY \\ UNIVERSITY OF WASHINGTON \\ AMERICAN MATHEMATICAL SOCIETY
}

The Supporting Institutions listed above contribute to the cost of publication of this Journal, but they are not owners or publishers and have no responsibility for its contents or policies.

Mathematical papers intended for publication in the Pacific Journal of Mathematics should be in typed form or offset-reproduced (not dittoed), double spaced with large margins. Underline Greek letters in red, German in green, and script in blue. The first paragraph or two must be capable of being used separately as a synopsis of the entire paper. Items of the bibliography should not be cited there unless absolutely necessary, in which case they must be identified by author and Journal, rather than by item number. Manuscripts, in duplicate, may be sent to any one of the four editors. Please classify according to the scheme of Math. Reviews, Index to Vol. 39. All other communications should be addressed to the managing editor, or Elaine Barth, University of California, Los Angeles, California, 90024.

100 reprints are provided free for each article, only if page charges have been substantially paid. Additional copies may be obtained at cost in multiples of 50 .

The Pacific Journal of Mathematics is issued monthly as of January 1966. Regular subscription rate: $\$ 72.00$ a year (6 Vols., 12 issues). Special rate: $\$ 36.00$ a year to individual members of supporting institutions.

Subscriptions, orders for back numbers, and changes of address should be sent to Pacific Journal of Mathematics, 103 Highland Boulevard, Berkeley, California, 94708.

PUBLISHED BY PACIFIC JOURNAL OF MATHEMATICS, A NON-PROFIT CORPORATION Printed at Jerusalem Academic Press, POB 2390, Jerusalem, Israel.

$$
\begin{gathered}
\text { Copyright (C) } 1975 \text { Pacific Journal of Mathematics } \\
\text { All Rights Reserved }
\end{gathered}
$$




\section{Pacific Journal of Mathematics}

\section{Vol. 58, No. $2 \quad$ April, 1975}

Zvi Artstein and John Allen Burns, Integration of compact set-valued functions . . . . . . . . . 297

Mark Benard, Characters and Schur indices of the unitary reflection group $[321]^{3} \ldots \ldots \ldots . .309$

Simeon M. Berman, A new characterization of characteristic functions of absolutely continuous

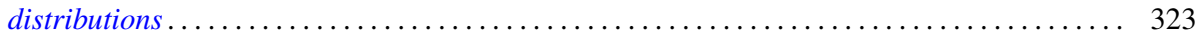

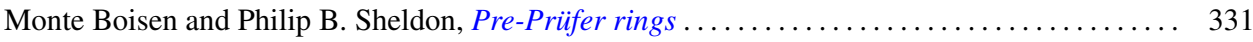

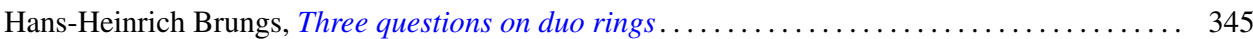

Iracema M. Bund, Birnbaum-Orlicz spaces of functions on groups................. 351

John D. Elwin and Donald R. Short, Branched immersions between 2-manifolds of higher

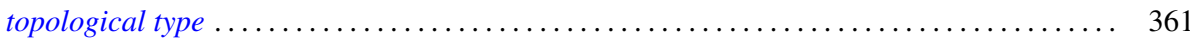

Eric Friedlander, Extension functions for rank 2, torsion free abelian groups . .......... 371

Jon Froemke and Robert Willis Quackenbush, The spectrum of an equational class of

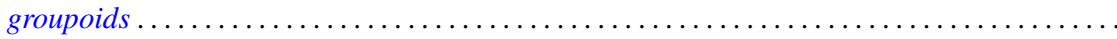

Barry J. Gardner, Radicals of supplementary semilattice sums of associative rings ...........

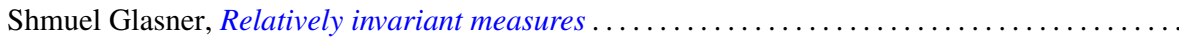

George Rudolph Gordh, Jr. and Sibe Mardesic, Characterizing local connectedness in inverse

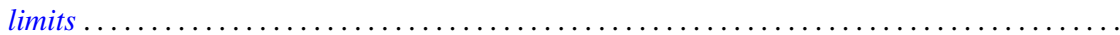

Siegfried Graf, On the existence of strong liftings in second countable topological spaces......

Stanley P. Gudder and D. Strawther, Orthogonally additive and orthogonally increasing

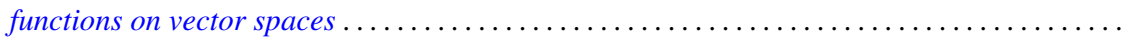

Darald Joe Hartfiel and Carlton James Maxson, A characterization of the maximal monoids and

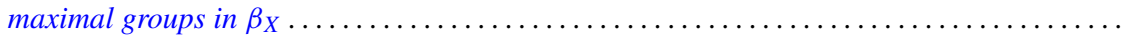

Robert E. Hartwig and S. Brent Morris, The universal flip matrix and the generalized faro-shuffle. .

William Emery Haver, Mappings between ANRs that are fine homotopy equivalences. .

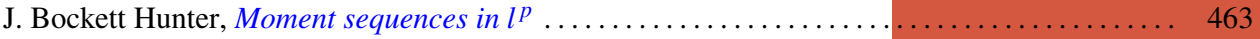

Barbara Jeffcott and William Thomas Spears, Semimodularity in the completion of a poset.... 467

Jerry Alan Johnson, A note on Banach spaces of Lipschitz functions . . . . . . . . . . . . 475

David W. Jonah and Bertram Manuel Schreiber, Transitive affine transformations on

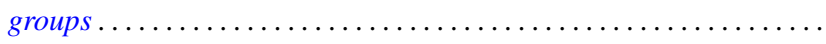

Karsten Juul, Some three-point subset properties connected with Menger's characterization of

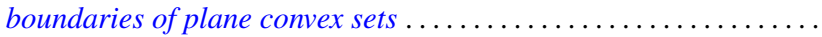

Ronald Brian Kirk, The Haar integral via non-standard analysis . . . . . . . . . . . . . 517

Justin Thomas Lloyd and William Smiley, On the group of permutations with countable

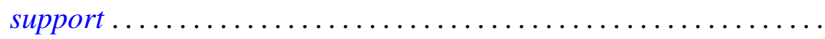

Erwin Lutwak, Dual mixed volumes .................................. 531

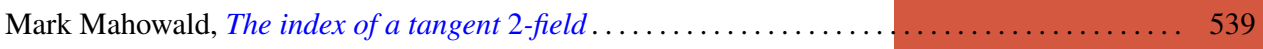

Keith Miller, Logarithmic convexity results for holomorphic semigroups . . . . . . . . . . . . 549

Paul Milnes, Extension of continuous functions on topological semigroups . . . . . . . . . . 553

Kenneth Clayton Pietz, Cauchy transforms and characteristic functions ................ 563

James Ted Rogers Jr., Whitney continua in the hyperspace $C(X) \ldots \ldots \ldots \ldots \ldots \ldots \ldots \ldots .569$

Jean-Marie G. Rolin, The inverse of a continuous additive functional . . . . . . . . . . . . 585

William Henry Ruckle, Absolutely divergent series and isomorphism of subspaces . ........ 605

Rolf Schneider, A measure of convexity for compact sets . ..................... 617

Alan Henry Schoenfeld, Continous measure-preserving maps onto Peano spaces .......... 627

V. Merriline Smith, Strongly superficial elements .......................... 643

Roger P. Ware, A note on quadratic forms over Pythagorean fields . . . . . . . . . . . . . . 651

Roger Allen Wiegand and Sylvia Wiegand, Finitely generated modules over Bezout rings . . . . 655

Martin Ziegler, A counterexample in the theory of definable automorphisms . . . . . . . . . 665 